University of Nebraska - Lincoln

DigitalCommons@University of Nebraska - Lincoln

Faculty Publications from Nebraska Center for

Materials and Nanoscience

Materials and Nanoscience, Nebraska Center

for $(\mathrm{NCMN})$

January 2007

\title{
Ultrasonic characterization of microstructure evolution during processing
}

Liyong Yang

University of Nebraska - Lincoln, lyang4@unl.edu

Joseph A. Turner

University of Nebraska - Lincoln, jaturner@unl.edu

Zheng Li

Department of Mechanics and Engineering Science, Peking University, Beijing 100871, China

Follow this and additional works at: https://digitalcommons.unl.edu/cmrafacpub

Part of the Nanoscience and Nanotechnology Commons

Yang, Liyong; Turner, Joseph A.; and Li, Zheng, "Ultrasonic characterization of microstructure evolution during processing" (2007). Faculty Publications from Nebraska Center for Materials and Nanoscience. 58. https://digitalcommons.unl.edu/cmrafacpub/58

This Article is brought to you for free and open access by the Materials and Nanoscience, Nebraska Center for (NCMN) at DigitalCommons@University of Nebraska - Lincoln. It has been accepted for inclusion in Faculty Publications from Nebraska Center for Materials and Nanoscience by an authorized administrator of DigitalCommons@University of Nebraska - Lincoln. 


\title{
Ultrasonic characterization of microstructure evolution during processing
}

\author{
Liyong Yang ${ }^{\text {a) }}$ and Joseph A. Turner \\ Department of Engineering Mechanics, W317.4 Nebraska Hall, University of Nebraska-Lincoln, \\ Lincoln, Nebraska 68588-0526 \\ Zheng Li \\ Department of Mechanics and Engineering Science, Peking University, Beijing 100871, China
}

(Received 12 June 2006; revised 27 September 2006; accepted 5 October 2006)

\begin{abstract}
Many cold-working processes for polycrystalline metals cause alignment of the grains with a single symmetry axis. This type of microstructure is called fiber texture. The existence of a preferred orientation of the grains has a significant influence on the propagation and scattering of ultrasonic waves, which are often used for material inspection. Knowledge of the wave attenuation of such textured materials is of both theoretical and practical interest to nondestructive testing and materials characterization. In this article, the quantitative relations between fiber texture and wave attenuations of hexagonal crystals are presented. The texture is characterized by a Gaussian distribution function that contains a single parameter that governs the transition of the texture from perfectly aligned crystals to statistically isotropic. Under this assumption, the materials of interest have a varying degree of transverse isotropy representative of processing conditions. Simple expressions for the attenuations of the three modes of waves are given in a concise, generalized representation. Finally, numerical results are presented and discussed in terms of the directional, frequency, and texture dependence. The results presented are expected to improve the understanding of the microstructure evolution during thermomechanical processing. (C) 2007 Acoustical Society of America. [DOI: 10.1121/1.2382749]
\end{abstract}

PACS number(s): 43.20.Bi, 43.20.Gp, 43.35.Cg [TDM]

Pages: $50-59$

\section{INTRODUCTION}

The macroscopic anisotropy of the physical properties of polycrystalline materials is generally determined by two factors, the anisotropy of the crystals, which is described by the single-crystal elastic constants, and the distribution of the crystals in space, which is called the texture. The simplest anisotropic symmetry class to be considered is that of medium with a single symmetry axis. The direction of the single symmetry axis is defined as the fiber direction. In this case, the fiber direction is not necessarily perpendicular to the sample surface, but typically is associated with processing conditions (e.g., rolling direction). The knowledge that the material microstructure directly affects the macroscopic material properties was a turning point in the field of materials manufacturing. Specific types of manufacturing processes are used to produce the corresponding microstructure in a controlled fashion. Many heat treatment processes, such as annealing, are used to relieve the internal stress state that develops during cold working, which allows the microstructure to rearrange itself to a state of lower energy. During such processing, individual crystals in a polycrystalline aggregate undergo orientation changes. Often, the recrystallization process creates material texture, or preferred orientation of grains. The degree and type of texture are best described quantitatively by the orientation distribution function (ODF).

\footnotetext{
a) Author to whom correspondence should be addressed; Electronic mail: lyang4@unl.edu
}

The distribution of the orientation occurring in the recrystallization textures of polycrystalline titanium and zinc may be represented by a Gaussian ODF (Li, 2000).

Ultrasonic techniques provide information about the interior microstructure due to the penetration of ultrasonic waves. In recent years, major advances in ultrasonic monitoring nondestructive evaluation (NDE) demonstrate a potential to characterize recrystallization processes. Previous analysis of wave propagation and scattering in polycrystals with fiber texture was focused mainly on samples with cubic crystal symmetry (Hirsekorn, 1985, 1986; Turner, 1999; Ahmed and Thompson, 1996). However, crystal symmetries other than cubic are also important. For example, the fiber texture of hexagonal crystals has been observed for a variety of materials, such as titanium, zinc, magnesium, ice, and many others.

The scattering of elastic waves by grains of polycrystals has received considerable attention. Contributions for cubic symmetry with uniformly distributed orientations of grains were made by Hirsekorn (1982, 1983), Stanke and Kino (1984), and Weaver (1990). The problem of wave propagation and scattering in the case of polycrystalline grains with an aligned [001] axis has been examined by Ahmed and Thompson (1996) and Turner (1999). In that case, the average medium is statistically transversely isotropic. Ahmed and Thompson $(1992,1996)$ also studied correlations defined by both equiaxed grains and grains with elongation. Most recently, wave attenuation in the case of orthorhombic-cubic symmetry was investigated by Yang and Turner (2004). 
In this article, quantitative relations between fiber texture and wave attenuations of hexagonal crystals are presented. The texture is characterized by a Gaussian distribution function that contains a single parameter that governs the transition of the texture from perfectly aligned grains to statistically isotropic. Under this assumption, the materials of interest have a varying degree of transverse isotropy representing various states of processing conditions. Simple expressions for the attenuations of the three modes of waves are given in a concise representation. The resulting attenuations are presented and discussed in terms of the directional, frequency, and texture dependence. The results presented are expected to improve the understanding of the microstructure evolution during thermomechanical processing. In addition, the present formulation may be used to study diffuse ultrasonic problems in a straightforward manner.

\section{EFFECTIVE ELASTIC STIFFNESS}

For textured materials, a detailed description of polycrystalline material properties in the sample requires a knowledge of the orientation distribution of all crystallites in the sample. The orientation of a given single crystallite is specified by the three Euler angles $\theta, \psi$, and $\varphi$. The orientation distribution of crystallite grains with preferred directions in the sample can be described by the orientation distribution function (ODF), $F(\theta, \psi, \varphi)$, which is the probability density function in terms of the three Euler angles. To discuss the orientation of a grain, a set of crystallite-fixed axes $X_{i}$ is chosen for a given grain. One may choose the sample-fixed axes $x_{i}$ in polycrystals. The crystallite axes $X_{i}$ and the sample axes $x_{i}$ are related through a rotation matrix using the three Euler angles. In general, the elastic modulus tensor for a single hexagonal crystallite is given by

$$
\begin{aligned}
c_{i j k l}= & \lambda^{h} \delta_{i j} \delta_{k l}+\mu^{h}\left(\delta_{i k} \delta_{j l}+\delta_{i l} \delta_{j k}\right)+A\left(\delta_{i j} \hat{e}_{k} \hat{e}_{l}+\delta_{k l} \hat{e}_{i} \hat{e}_{j}\right) \\
& +B\left(\delta_{i k} \hat{e}_{j} \hat{e}_{l}+\delta_{i l} \hat{e}_{j} \hat{e}_{k}+\delta_{j k} \hat{e}_{i} \hat{e}_{l}+\delta_{j l} \hat{e}_{i} \hat{e}_{k}\right)+D \hat{e}_{i} \hat{e}_{j} \hat{e}_{k} \hat{e}_{l},
\end{aligned}
$$

where the unit vector $\hat{\mathbf{e}}$ is defined as the crystal sixfold symmetry axis. The five coefficients in Eq. (1) are given in terms of single hexagonal crystallite elastic constants $c_{11}, c_{33}, c_{44}$, $c_{12}, c_{13}, c_{66}$ as

$$
\begin{aligned}
& \lambda^{h}=c_{12}=c_{11}-2 c_{66}, \quad \mu^{h}=c_{66}, \\
& A=c_{13}-c_{12}, \quad B=c_{44}-c_{66}, \\
& D=c_{11}+c_{33}-2 c_{13}-c_{44} .
\end{aligned}
$$

The average medium is characterized by the average stiffness tensor. If the average elastic stiffness tensor, $\langle\mathbf{c}\rangle$, represents a transversely isotropic medium, it may be written as a function of Kronecker deltas and the unit vector $\hat{\mathbf{n}}$, which defines the fiber direction (or uniaxial symmetry axis). It can be expressed by

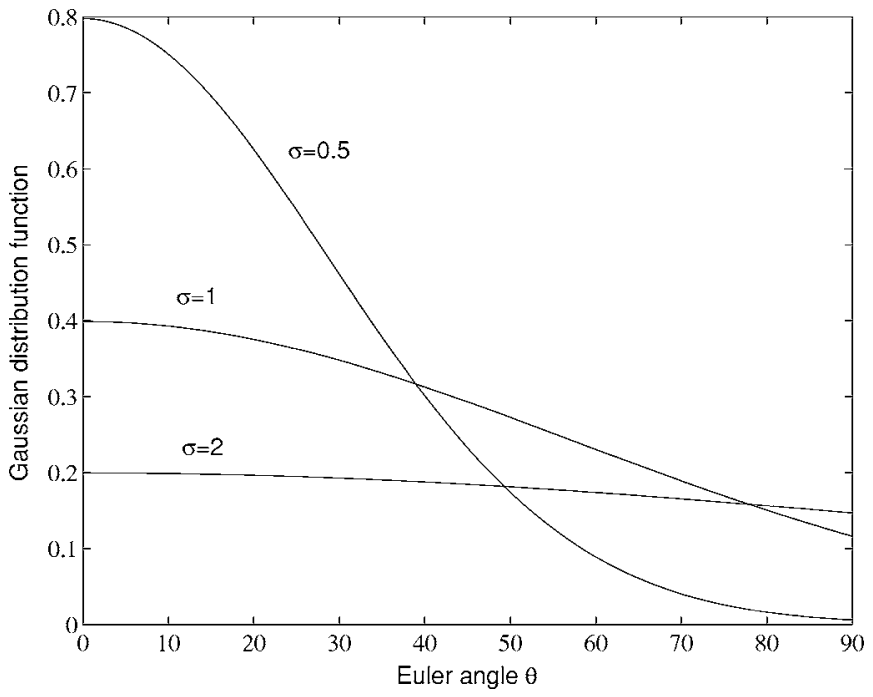

FIG. 1. Gaussian distribution function with various parameters $\sigma$.

$$
\begin{aligned}
C_{i j k l}= & \left\langle c_{i j k l}\right\rangle=\Gamma_{1} \delta_{i j} \delta_{k l}+\Gamma_{2}\left(\delta_{i k} \delta_{j l}+\delta_{i l} \delta_{j k}\right) \\
& +\Gamma_{3}\left(\delta_{i j} \hat{n}_{k} \hat{n}_{l}+\delta_{k l} \hat{n}_{i} \hat{n}_{j}\right)+\Gamma_{4}\left(\delta_{i k} \hat{n}_{j} \hat{n}_{l}+\delta_{i l} \hat{n}_{j} \hat{n}_{k}\right. \\
& \left.+\delta_{j k} \hat{n}_{i} \hat{n}_{l}+\delta_{j l} \hat{n}_{i} \hat{n}_{k}\right)+\Gamma_{5} \hat{n}_{i} \hat{n}_{j} \hat{n}_{k} \hat{n}_{l} \\
= & \Gamma_{1} J_{1}+\Gamma_{2} J_{2}+\Gamma_{3} J_{3}+\Gamma_{4} J_{4}+\Gamma_{4} J_{5},
\end{aligned}
$$

where Eq. (3) serves to define the tensors $J_{i}$ with coefficients $\Gamma_{i}(i=1,2,3,4,5)$. Relations between $\Gamma_{i}$ and $C_{i j k l}$ are derived below. The ensemble average stiffness of the medium is defined explicitly by

$\left\langle c_{i j k l}\right\rangle=\frac{1}{8 \pi^{2}} \int_{0}^{\pi} \int_{0}^{2 \pi} \int_{0}^{2 \pi} c_{i j k l} F(\sigma, \theta, \psi, \varphi) \sin \theta d \psi d \varphi d \theta$,

where $c_{i j k l}$ is given in Eq. (1).

As discussed above, an orientation distribution function (ODF) $F(\sigma, \theta, \psi, \varphi)$ is introduced to represent uniquely the crystallite orientation distribution. Here, a Gaussian distribution function is adopted as the ODF, which is a reasonable approximation for the fiber texture associated with hexagonal materials $(\mathrm{Li}, 2000)$. That is, the crystallite distribution depends only on Euler angle $\theta$ and is independent of angles $\varphi$ and $\psi$,

$$
F(\sigma, \theta)=F_{0} \exp \left(-\frac{\theta^{2}}{2 \sigma^{2}}\right)
$$

where $\sigma$ is a single parameter that governs the transition of the texture from perfectly aligned grains to statistically isotropic. The normalization coefficient $F_{0}$ is defined by $\left(1 / 8 \pi^{2}\right) \int_{0}^{\pi} \int_{0}^{2 \pi} \int_{0}^{2 \pi} F(\sigma, \theta, \psi, \varphi) \sin \theta d \psi d \varphi d \theta=1$. Thus, it may be expressed as

$$
\begin{aligned}
F_{0}= & \frac{\sqrt{\pi} \sigma}{4 \sqrt{2}} \exp \left(-\frac{\sigma^{2}}{2}\right)\left[2 \operatorname{Erfi}\left(\frac{\sigma}{\sqrt{2}}\right)-\operatorname{Erfi}\left(\frac{-i \pi+\sigma^{2}}{\sqrt{2} \sigma}\right)\right. \\
& \left.-\operatorname{Erfi}\left(\frac{i \pi+\sigma^{2}}{\sqrt{2} \sigma}\right)\right],
\end{aligned}
$$

where Erfi is the imaginary error function. The shape of this type of distribution function for several values of $\sigma$ is shown 
in Fig. 1. Two extreme cases are observed. One is a quasisingle crystal with perfectly aligned grains which occurs as $\sigma \rightarrow 0$. The other limiting case is a statistically isotropic polycrystal with randomly oriented grains which occurs as $\sigma \rightarrow \infty$. Other degrees of texture between these limits can be realized by varying $\sigma$. Thus, such a distribution function allows a single parameter to model this transition.

In order to obtain the coefficients of $\Gamma_{i}$, the following identities are needed:

$$
\begin{aligned}
\left\langle\hat{e}_{i} \hat{e}_{j}\right\rangle=\delta_{i j}\left[\frac{1}{2}\left(I_{\sigma}^{0}-I_{\sigma}^{2}\right)\right]+\hat{n}_{i} \hat{n}_{j}\left[\frac{1}{2}\left(-I_{\sigma}^{0}+3 I_{\sigma}^{2}\right)\right], & \\
\left\langle\hat{e}_{i} \hat{e}_{j} \hat{e}_{k} \hat{e}_{l}\right\rangle= & \left(J_{1}+J_{2}-J_{3}-J_{4}+3 J_{5}\right)\left[\frac{1}{8}\left(I_{\sigma}^{0}-2 I_{\sigma}^{2}+I_{\sigma}^{4}\right)\right] \\
& +\left(J_{5}\right) I_{\sigma}^{4}+\left(J_{3}+J_{4}-6 J_{5}\right)\left[\frac{1}{2}\left(I_{\sigma}^{2}-I_{\sigma}^{4}\right)\right],
\end{aligned}
$$

where the coefficients $I_{\sigma}^{m}(m=0,2,4,6,8)$ are defined by

$$
I_{\sigma}^{m}=\frac{1}{2} \int_{0}^{\pi} F(\sigma, \theta) \cos ^{m} \theta \sin \theta d \theta .
$$

Using the identities given in Eq. (7), the five coefficients $\Gamma_{i}$ in Eq. (3) are obtained and expressed as

$$
\begin{aligned}
& \Gamma_{1}=\lambda^{h}+2 A M_{1}+D M_{3}, \\
& \Gamma_{2}=\mu^{h}+2 B M_{1}+D M_{3}, \\
& \Gamma_{3}=A M_{2}-D M_{3}+D M_{5}, \\
& \Gamma_{4}=B M_{2}-D M_{3}+D M_{5}, \\
& \Gamma_{5}=3 D M_{3}+D M_{4}-6 D M_{5},
\end{aligned}
$$

where $M_{i}$ are expressed in terms of the coefficients in Eq. (8) as

$$
\begin{aligned}
& M_{1}=\frac{1}{2}\left(I_{\sigma}^{0}-I_{\sigma}^{2}\right), \quad M_{2}=\frac{1}{2}\left(-I_{\sigma}^{0}+3 I_{\sigma}^{2}\right), \quad M_{5}=I_{\sigma}^{4}, \\
& M_{3}=\frac{1}{8}\left(I_{\sigma}^{0}-2 I_{\sigma}^{2}+I_{\sigma}^{4}\right), \quad M_{4}=\frac{1}{2}\left(I_{\sigma}^{2}-I_{\sigma}^{4}\right) .
\end{aligned}
$$

Hence, the five effective elastic constants of the ensemble average medium are given by

$$
\begin{aligned}
& C_{11}=\Gamma_{1}+2 \Gamma_{2}, \quad C_{33}=\Gamma_{1}+2 \Gamma_{2}+2 \Gamma_{3}+4 \Gamma_{4}+\Gamma_{5}, \\
& C_{44}=\Gamma_{2}+\Gamma_{4}, \quad C_{12}=\Gamma_{1}, \quad C_{13}=\Gamma_{1}+\Gamma_{3}, \\
& C_{66}=\frac{1}{2}\left(C_{11}-C_{12}\right) .
\end{aligned}
$$

It is known that the identities given in Eq. (7) can be simplified under the two limiting cases of interest as (Fedorov, 1968)

$$
\left\langle\hat{e}_{i} \hat{e}_{j}\right\rangle=\frac{1}{3} \delta_{i j}, \quad\left\langle\hat{e}_{i} \hat{e}_{j} \hat{e}_{k} \hat{e}_{l}\right\rangle=\frac{1}{15}\left(J_{1}+J_{2}\right), \quad \text { when } \sigma \rightarrow \infty,
$$

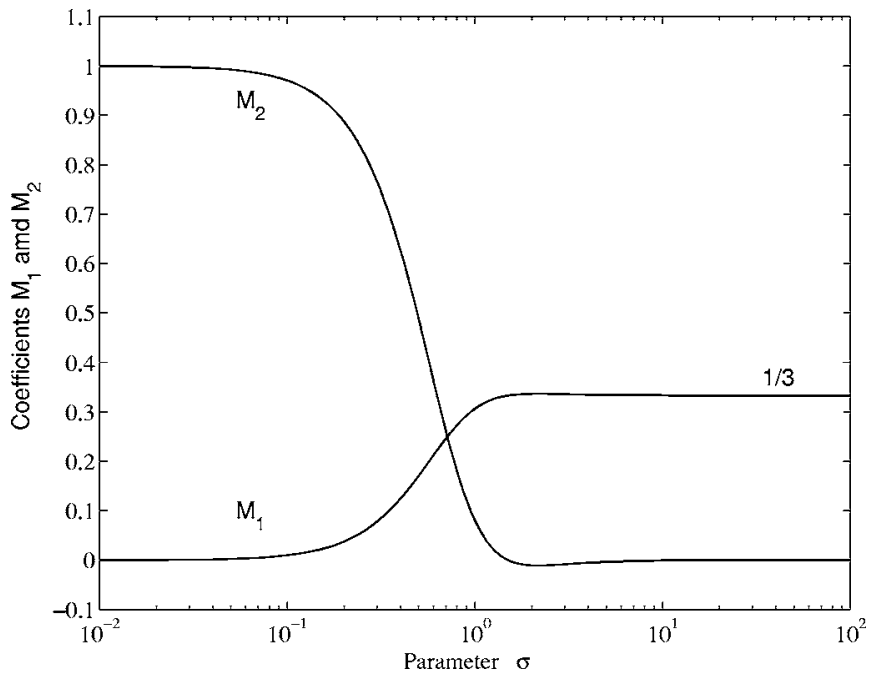

FIG. 2. Coefficients $M_{1}$ and $M_{2}$ as a function of texture parameter $\sigma$.

$$
\text { and }\left\langle\hat{e}_{i} \hat{e}_{j}\right\rangle=\hat{n}_{i} \hat{n}_{j}, \quad\left\langle\hat{e}_{i} \hat{e}_{j} \hat{e}_{k} \hat{e}_{l}\right\rangle=\hat{n}_{i} \hat{n}_{j} \hat{n}_{k} \hat{n}_{l}, \quad \text { when } \sigma \rightarrow 0 \text {. }
$$

The identities in Eq. (7) may be rewritten as

$$
\begin{aligned}
& \left\langle\hat{e}_{i} \hat{e}_{j}\right\rangle=M_{1} \delta_{i j}+M_{2} \hat{n}_{i} \hat{n}, \\
& \left\langle\hat{e}_{i} \hat{e}_{j} \hat{e}_{k} \hat{e}_{l}\right\rangle=S_{1}\left(J_{1}+J_{2}\right)+S_{2}\left(J_{3}+J_{4}\right)+S_{3} J_{5},
\end{aligned}
$$

where

$$
\begin{aligned}
& S_{1}=\frac{1}{8}\left(I_{\sigma}^{0}-2 I_{\sigma}^{2}+I_{\sigma}^{4}\right), \\
& S_{2}=\frac{1}{8}\left(-I_{\sigma}^{0}+6 I_{\sigma}^{2}-5 I_{\sigma}^{4}\right), \quad S_{3}=\frac{1}{8}\left(3 I_{\sigma}^{0}-30 I_{\sigma}^{2}+35 I_{\sigma}^{4}\right) .
\end{aligned}
$$

The five coefficients $M_{1}$ and $M_{2}$, and $S_{1}, S_{2}$, and $S_{3}$ are shown in Figs. 2 and 3, respectively. It is observed as $\sigma$ $\rightarrow \infty$, the coefficients $M_{2}$, and $S_{2}$ and $S_{3}$ approach zero, and as $\sigma \rightarrow 0$, the coefficients $M_{1}$, and $S_{1}$ and $S_{2}$ approach zero. Thus, the expected limiting behavior for $\mathbf{C}$ is observed. For the statistically isotropic case, the average elastic stiffness of a hexagonal crystallite may be simplified as ( $\mathrm{Li}$ and Thompson, 1990)

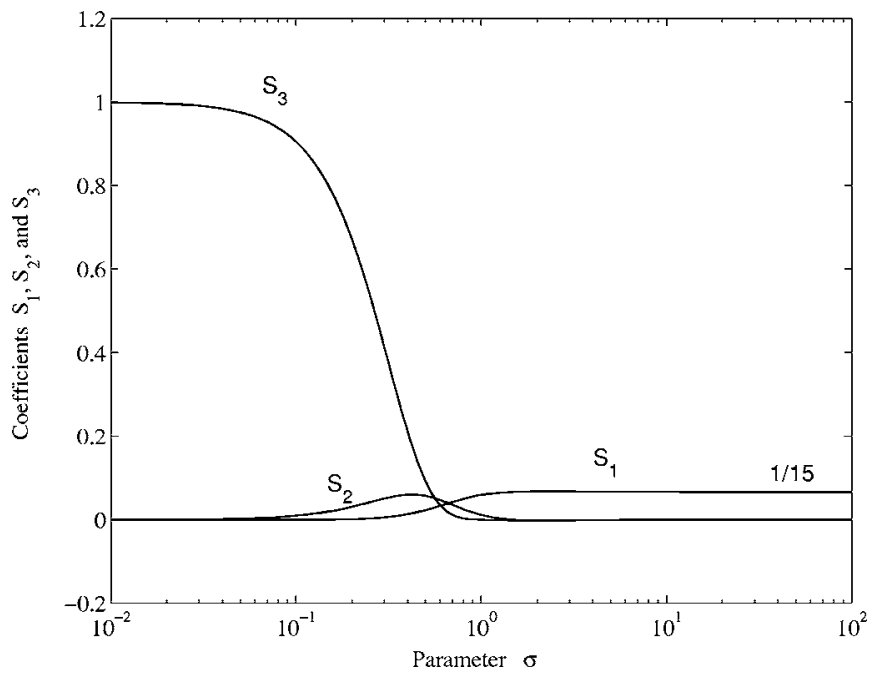

FIG. 3. Coefficients $S_{1}, S_{2}$, and $S_{3}$ as a function of texture parameter $\sigma$. 


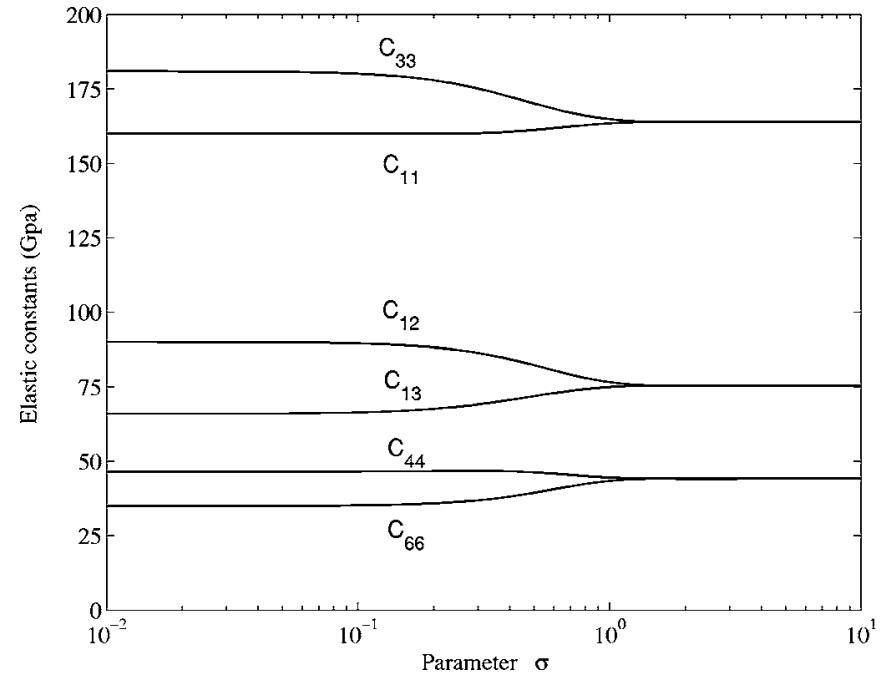

FIG. 4. Effective elastic constants as a function of texture parameter $\sigma$.

$$
\begin{aligned}
C_{i j k l}= & \bar{\lambda} \delta_{i j} \delta_{k l}+\bar{\mu}\left(\delta_{i k} \delta_{j l}+\delta_{i l} \delta_{j k}\right) \\
= & \left(\lambda^{h}+\frac{2}{3} A+\frac{D}{15}\right) \delta_{i j} \delta_{k l}+\left(\mu^{h}+\frac{2}{3} B+\frac{D}{15}\right) \\
& \times\left(\delta_{i k} \delta_{j l}+\delta_{i l} \delta_{j k}\right) .
\end{aligned}
$$

Example results of the effective elastic constants are now shown for a titanium polycrystal. The material constants of the single crystallite used here are $c_{11}=160 \mathrm{GPa}, c_{12}$ $=90 \mathrm{GPa}, c_{13}=66 \mathrm{GPa}, c_{33}=181 \mathrm{GPa}$, and $c_{44}=46.5 \mathrm{GPa}$. In Fig. 4, the elastic constants are presented as a function of $\sigma$, where $\sigma$ governs the crystal alignment from perfectly aligned $(\sigma=0)$ to randomly aligned $(\sigma \rightarrow \infty)$. It is clearly seen from Fig. 4 that as $\sigma$ approaches zero, the grains in polycrystals become perfectly aligned. In this case, the polycrystal behaves as a quasi-single crystal, the elastic constants of which reduce to the appropriate single-crystal constants. When $\sigma$ approaches infinity, the grains in the polycrystal are randomly oriented as expected. In such a case, the five independent elastic constants of the polycrystal reduce to two independent elastic constants. The transition between these two limits is seen clearly. Because the focus here is on wave propagation and scattering phenomena, the directional dependence of the wave speeds is of importance. Thus, slowness surfaces are presented for various parameter $\sigma$ for the shear horizontal, quasilongitudinal, and quasishear waves, respectively, in Figs. 5, 6, and 7. The transition of the texture from perfectly aligned to statistically isotropic is clear.

\section{ATTENUATION}

The scattering of elastic waves, often characterized by the attenuation, in a polycrystal results from the misalignment of the grains. To calculate the attenuations, the relevant inner products on the covariance of the moduli fluctuations are required (Turner, 1999). These may be written as

$$
\Xi_{\cdots \cdots \hat{\mathbf{u}} \hat{\mathbf{p}} \hat{\mathbf{p}} \hat{\mathbf{s}} \hat{\mathbf{v}}}=\Xi_{\alpha \beta \gamma \delta}^{i j k l} \hat{u}_{\beta} \hat{u}_{k} \hat{p}_{\alpha} \hat{p}_{l} \hat{s}_{i} \hat{s}_{\delta} \hat{v}_{\gamma} \hat{v}_{j}
$$

where the covariance of the moduli fluctuations $\Xi_{\alpha \beta \gamma \delta}^{i j k l}$ is given explicitly by

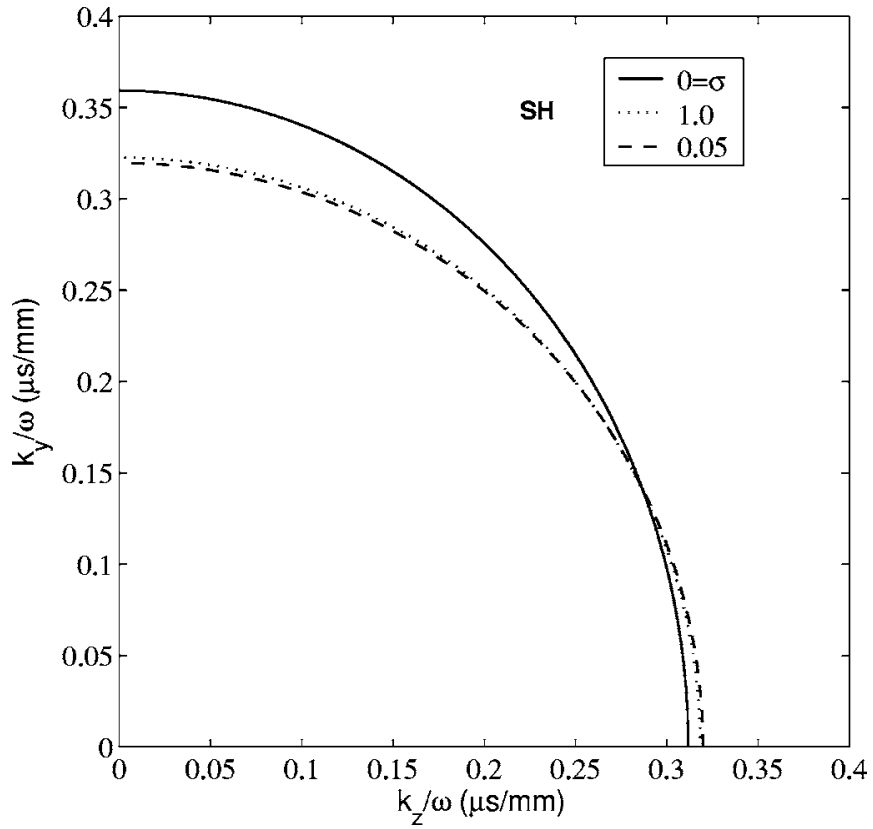

FIG. 5. Slowness surface of the shear horizontal wave $(\mathrm{SH})$.

$$
\Xi_{i j k l}^{\alpha \beta \gamma \delta}=\left\langle c_{i j k l} c_{\alpha \beta \gamma \delta}\right\rangle-\left\langle c_{i j k l}\right\rangle\left\langle c_{\alpha \beta \gamma \delta}\right\rangle .
$$

The brackets, \langle\rangle , denote an ensemble average over all orientations of grains. The average elastic stiffness tensor, $\langle\mathbf{c}\rangle$, is given in Eq. (4) for the case of interest here. The first term in Eq. (15) $\langle\mathbf{c c}\rangle$ is defined by

$$
\begin{aligned}
\left\langle c_{i j k l} c_{\alpha \beta \gamma \delta}\right\rangle= & \frac{1}{8 \pi^{2}} \int_{0}^{\pi} \int_{0}^{2 \pi} \int_{0}^{2 \pi} c_{i j k l} c_{\alpha \beta \gamma \delta} F(\sigma, \theta) \\
& \times \sin \theta d \psi d \varphi d \theta .
\end{aligned}
$$

In order to carry out the calculations in Eq. (16), the following general identities are given:

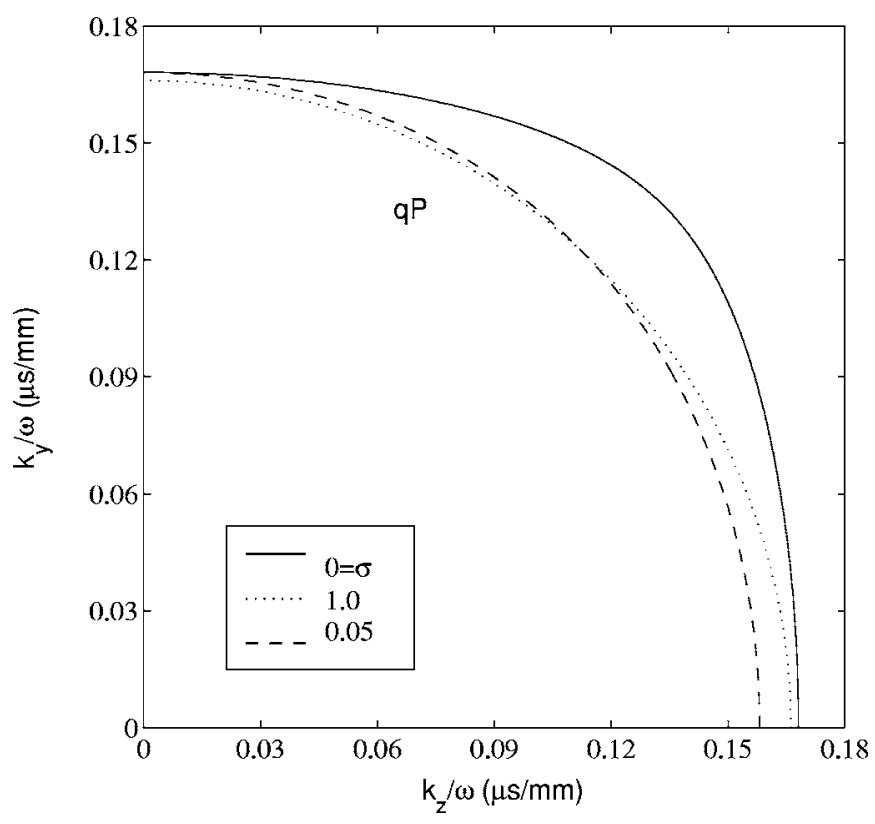

FIG. 6. Slowness surface of the quasilongitudinal wave $(q P)$. 


$$
\begin{aligned}
&\left\langle\hat{e}_{i} \hat{e}_{j} \hat{e}_{k} \hat{e}_{l} \hat{e}_{\alpha} \hat{e}_{\beta}\right\rangle=\left[\frac{1}{48}\left(I_{\sigma}^{0}-3 I_{\sigma}^{2}+3 I_{\sigma}^{4}-I_{\sigma}^{6}\right)\right]\left\{\Delta_{i j} \Delta_{k l} \Delta_{\alpha \beta}+\text { all permutations-15 terms in all }\right\} \\
&+\left[\frac{1}{8}\left(I_{\sigma}^{2}-2 I_{\sigma}^{4}+I_{\sigma}^{6}\right)\right]\left\{\hat{n}_{i} \hat{n}_{j}\left[\Delta_{k l} \Delta_{\alpha \beta}+\Delta_{k \beta} \Delta_{l \alpha}+\Delta_{k \alpha} \Delta_{l \beta}\right]+\text { all permutations-15 terms in all }\right\} \\
&+\left[\frac{1}{2}\left(I_{\sigma}^{4}-I_{\sigma}^{6}\right)\right]\left\{\hat{n}_{i} \hat{n}_{j} \hat{n}_{k} \hat{n}_{l} \Delta_{i j}+\text { all permutations-15 terms in all }\right\}+\left[I_{\sigma}^{6}\right] \hat{n}_{i} \hat{n}_{j} \hat{n}_{k} \hat{n}_{l} \hat{n}_{\alpha} \hat{n}_{\beta}, \\
&\left\langle\hat{e}_{i} \hat{e}_{j} \hat{e}_{k} \hat{e}_{l} \hat{e}_{\alpha} \hat{e}_{\beta} \hat{e}_{\gamma} \hat{e}_{\delta}\right\rangle= {\left[\frac{1}{384}\left(I_{\sigma}^{0}-4 I_{\sigma}^{2}+6 I_{\sigma}^{4}-4 I_{\sigma}^{6}+I_{\sigma}^{8}\right)\right]\left\{\Delta_{i j} \Delta_{k l} \Delta_{\alpha \beta} \Delta_{\gamma \delta}+\text { all permutations-105 terms in all }\right\} } \\
&+\left[\frac{1}{48}\left(I_{\sigma}^{2}-3 I_{\sigma}^{4}+3 I_{\sigma}^{6}-I_{\sigma}^{8}\right)\right]\left\{\hat{n}_{i} \hat{n}_{j} \Delta_{k l} \Delta_{\alpha \beta} \Delta_{\gamma \delta}+\text { all permutations-420 terms in all }\right\} \\
&+\left[\frac{1}{8}\left(I_{\sigma}^{4}-2 I_{\sigma}^{6}+I_{\sigma}^{8}\right)\right]\left\{\hat{n}_{i} \hat{n}_{j} \hat{n}_{k} \hat{n}_{l} \Delta_{\alpha \beta} \Delta_{\gamma \delta}+\text { all permutations-210 terms in all }\right\} \\
&+\left[\frac{1}{2}\left(I_{\sigma}^{6}-I_{\sigma}^{8}\right)\right]\left\{\hat{n}_{i} \hat{n}_{j} \hat{n}_{k} \hat{n}_{l} \hat{n}_{\alpha} \hat{n}_{\beta} \Delta_{\gamma \delta}+\text { all permutations-28 terms in all }\right\}+\left[I_{\sigma}^{8}\right] \hat{n}_{i} \hat{n}_{j} \hat{n}_{k} \hat{n}_{l} \hat{n}_{\alpha} \hat{n}_{\beta} \hat{n}_{\gamma} \hat{n}_{\delta},
\end{aligned}
$$

where $\Delta_{m n}=\delta_{m n}-\hat{n}_{m} \hat{n}_{n}$. In Fig. 8, examples of a few terms of $\langle\mathbf{c c}\rangle$ are shown. As expected those values reach a constant value, respectively, as $\sigma \rightarrow \infty$. Using the identities given in Eqs. (7), (17), and (18), the covariance $\Xi_{i j k l}^{\alpha \beta \gamma \delta}$ can be written in a general form, which is not presented here due to brevity. Example values of the covariance $\Xi_{i j k l}^{\alpha \beta \gamma \delta}$ of interest are plotted in Fig. 9. It is observed from Fig. 9 that the covariance $\Xi_{i j k l}^{\alpha \beta \gamma \delta}$ reduces to two constants when $\sigma$ becomes larger.

For the inner products $\Xi_{\cdots \cdots \hat{\mathbf{u}} \hat{\mathbf{p}} \hat{\mathbf{s}} \hat{\mathbf{v}} \mathbf{v}}$ presented in Eq. (14), the vectors $\hat{\mathbf{p}}$ and $\hat{\mathbf{s}}$, respectively, represent the incoming and outgoing propagation directions. The vectors $\hat{\mathbf{u}}$ and $\hat{\mathbf{v}}$ are vectors defining the polarization directions of the particular waves. Without loss of generality, the vectors are defined

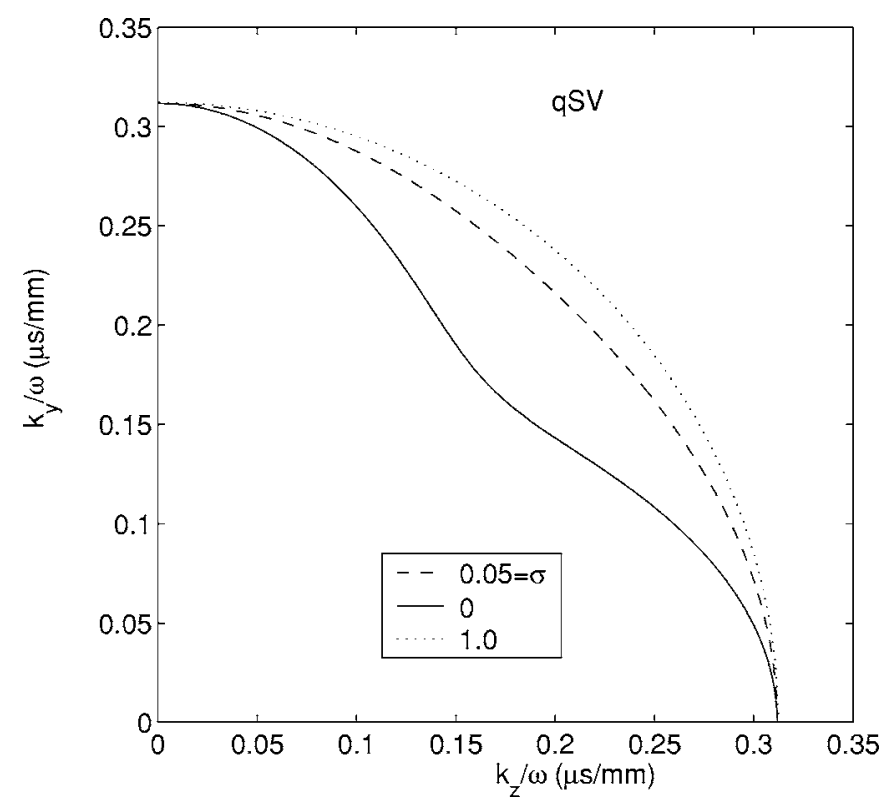

FIG. 7. Slowness surface of the quasi-shear vertical wave $(q S V)$. with respect to a general $x y z$ coordinate system as shown in Fig. 10. The vectors $\hat{\mathbf{n}}, \hat{\mathbf{p}}$, and $\hat{\mathbf{s}}$ are given by

$$
\begin{aligned}
& \hat{\mathbf{n}}=\hat{\mathbf{z}}, \quad \hat{\mathbf{p}}=\hat{\mathbf{x}} \sin \Theta+\hat{\mathbf{y}} \cos \Theta, \\
& \hat{\mathbf{s}}=\hat{\mathbf{x}} \quad \sin \Theta^{\prime} \cos \phi^{\prime}+\hat{\mathbf{y}} \sin \Theta^{\prime} \sin \phi^{\prime}+\hat{\mathbf{z}} \cos \Theta^{\prime} .
\end{aligned}
$$

The polarization vectors $\hat{\mathbf{u}}$ and $\hat{\mathbf{v}}$ as shown in Fig. 10 are given elsewhere (Turner, 1999). Substituting those unit vectors into Eq. (14), and combining with Eq. (15), the general inner products $\Xi_{\ldots}^{\cdots} \hat{\text { ûp } \hat{p} \hat{s} \hat{v} \hat{v}}$ may be calculated. An example inner product associated with the attenuation of a shear horizontal wave is presented in the Appendix. If the tensorial and spatial components of covariance are assumed to be independent, the spatial correlation function $W$ is not dependent on the tensorial part. For simplicity, the correlation function $W$ is assumed to have an exponential form

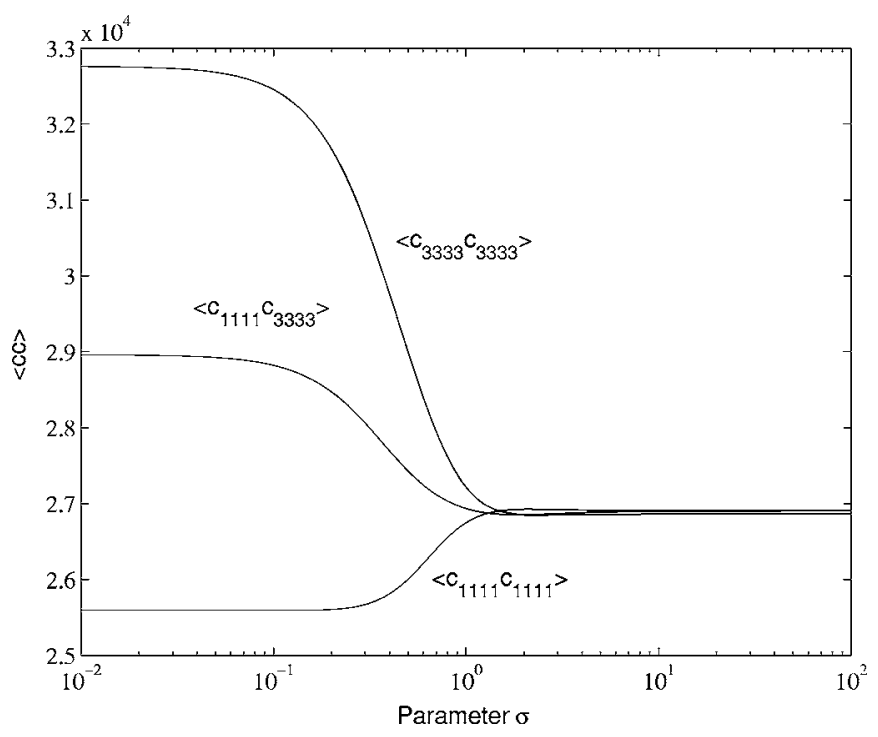

FIG. 8. Various terms $\langle\mathbf{c c}\rangle$ as a function of $\sigma$.

Yang et al.: Microstructure evolution during processing 


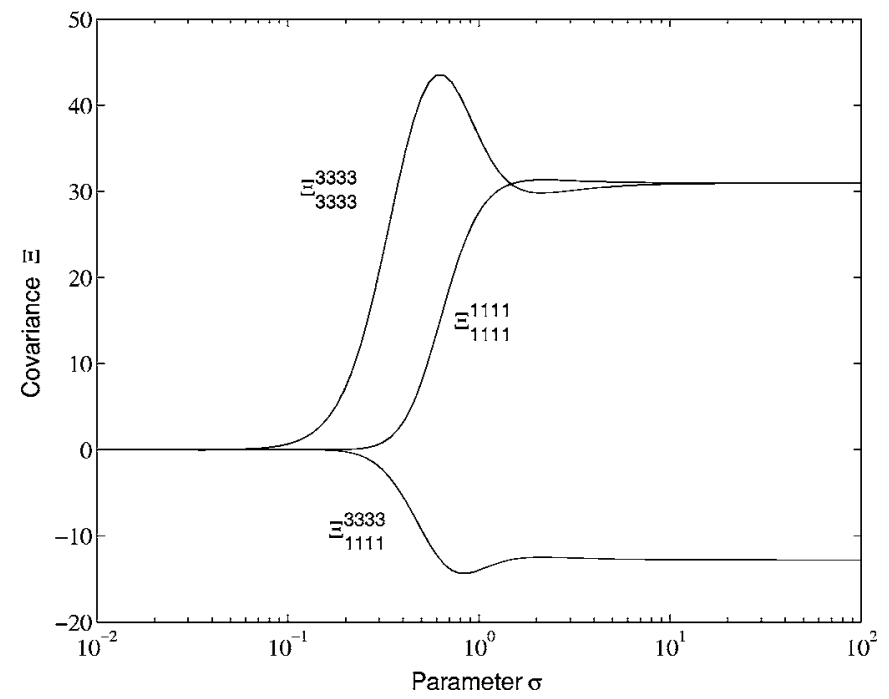

FIG. 9. Covariance $\Xi$ as a function of $\sigma$.

$$
W(r)=e^{-r / L},
$$

where $L$ is the spatial correlation length, which is of the order of the grain radius of the crystallites. In general, the simple exponential form of the correlation function must be

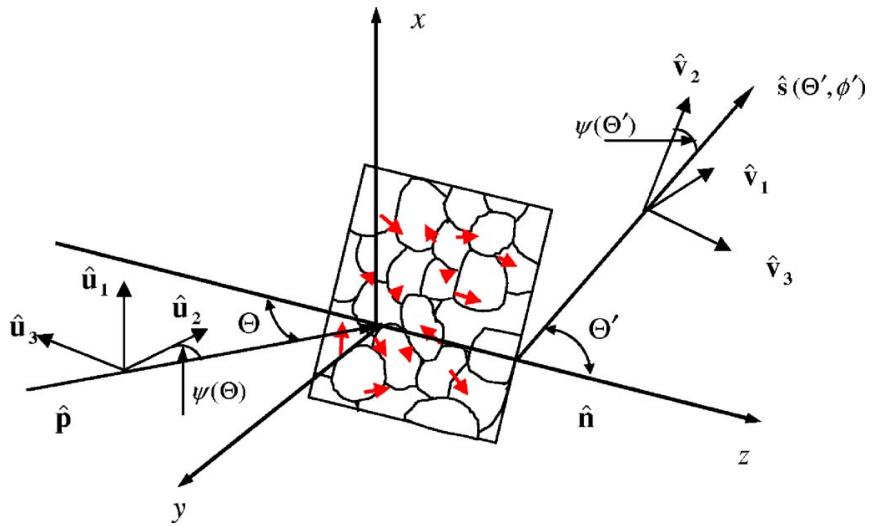

FIG. 10. (Color online) Geometry for the propagation direction $\hat{\mathbf{p}}$, the scattered direction $\hat{\mathbf{s}}$, and the respective polarization directions $\hat{\mathbf{u}}$ and $\hat{\mathbf{v}}$.

modified for the polycrystalline materials with texture since grain elongation often exists. A more general expression (Ahmed and Thompson, 1992) will be used in future work. The influence of the choice of correlation function on the attenuations is not the subject of the present work.

Using the expressions given above, the resulting dimensionless attenuations are written in a general form as (Yang and Turner, 2004)

$$
\begin{aligned}
& \alpha_{\beta}(\hat{\mathbf{p}}) L=\frac{x_{\beta}^{4} c_{\beta}(\hat{\mathbf{p}})}{2 \rho^{2}}\left\{\int_{4 \pi} \frac{\Xi_{\cdots \cdots \hat{\mathbf{u}}_{K} \hat{\mathbf{p}} \hat{\mathbf{p}} \hat{\mathbf{v}}_{1}}\left(\hat{\mathbf{p}} \hat{\mathbf{v}}_{1}(\hat{\mathbf{p}}, \hat{\mathbf{s}})\right.}{\left(1+x_{\beta}^{2}(\hat{\mathbf{p}})+x_{S H}^{2}(\hat{\mathbf{s}})-2 x_{\beta}(\hat{\mathbf{p}}) x_{S H}(\hat{\mathbf{s}}) \hat{\mathbf{p}} \cdot \hat{\mathbf{s}}\right)^{2}} \frac{1}{c_{S H}^{5}(\hat{\mathbf{s}})} d^{2} \hat{s}\right. \\
& +\int_{4 \pi} \frac{\exists \hat{\mathbf{u}}_{N} \hat{\hat{\mathbf{s}}} \hat{\mathbf{v}}_{2}(\hat{\mathbf{p}}, \hat{\mathbf{s}})}{\left(1+x_{\beta}^{2}(\hat{\mathbf{p}})+x_{q P}^{2}(\hat{\mathbf{s}})-2 x_{\beta}(\hat{\mathbf{p}}) x_{q P}(\hat{\mathbf{s}}) \hat{\mathbf{p}} \cdot \hat{\mathbf{s}}\right)^{2}} \frac{1}{c_{q P}^{5}(\hat{\mathbf{s}})} d^{2} \hat{s} \\
& \left.+\int_{4 \pi} \frac{\Xi_{\cdots}^{\cdots} \hat{\mathbf{u}}_{K} \hat{\mathbf{p}} \hat{\mathbf{s}} \hat{\mathbf{v}}_{3}}{\left(1+x_{\beta}^{2}(\hat{\mathbf{p}})+x_{q S V}^{2}(\hat{\mathbf{s}})-2 x_{\beta}(\hat{\mathbf{p}}) x_{q S V}(\hat{\mathbf{s}})\right)} \frac{1}{\left.\hat{\mathbf{p}}_{\mathbf{p}} \cdot \hat{\mathbf{s}}\right)^{2}} \frac{1}{c_{q S V}^{5}(\hat{\mathbf{s}})} d^{2} \hat{s}\right\},
\end{aligned}
$$

where $K$ is defined as the polarization for the wave type $\beta(1$, 2 , or 3 for wave types $S H, q P$, and $q S V$, respectively). The normalized frequency $x_{\beta}$ is defined by $x_{\beta}=\omega L / c_{\beta}$, where $\omega$ is the frequency and $c_{\beta}$ is the wave velocity of each type. In Eq. (20), it can be seen that the integrals are over the unit sphere, which is defined by unit vector $\hat{\mathbf{s}}$. Further details of the scattering model can be reviewed by the reader in the articles of Weaver (1990) and Turner (1999). For the extreme case of statistical isotropy $(\sigma \rightarrow \infty)$, the dimensionless longitudinal and transverse attenuations can be given as follows:

$$
\alpha_{L}=\alpha_{L L} L+\alpha_{L T} L, \quad \alpha_{T}=\alpha_{T L} L+\alpha_{T T} L,
$$

where

$\alpha_{L L} L=\frac{x_{L}^{4}}{2 c_{L}^{4}} \int_{-1}^{+1} \frac{\Xi \ldots \ldots \hat{\mathbf{p}} \hat{\mathbf{p}} \hat{\mathbf{s}} \hat{\mathbf{s}} \mathbf{s}}{\left[1+2 x_{L}^{2}(1-\chi)\right]^{2}} d \chi$

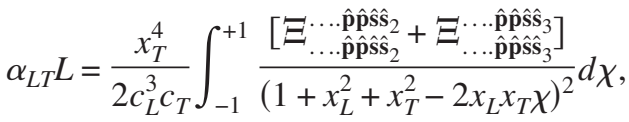

$$
\begin{aligned}
& \alpha_{T L} L=\frac{1}{2}\left(\frac{c_{T}}{c_{L}}\right)^{2} \alpha_{L T} L \\
& \alpha_{T T} L=\frac{x_{T}^{4}}{4 c_{T}^{4}}
\end{aligned}
$$

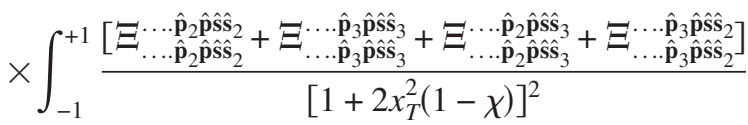

$$
\begin{aligned}
& \times d \chi \text {, }
\end{aligned}
$$

with $\chi=\hat{\mathbf{p}} \cdot \hat{\mathbf{s}}=\cos \Phi$, where $\Phi$ is the angle separating the propagation direction $\hat{\mathbf{p}}$ and the scattering direction $\hat{\mathbf{s}}$. The detailed expressions for the inner products in Eq. (22) are given in the Appendix. 


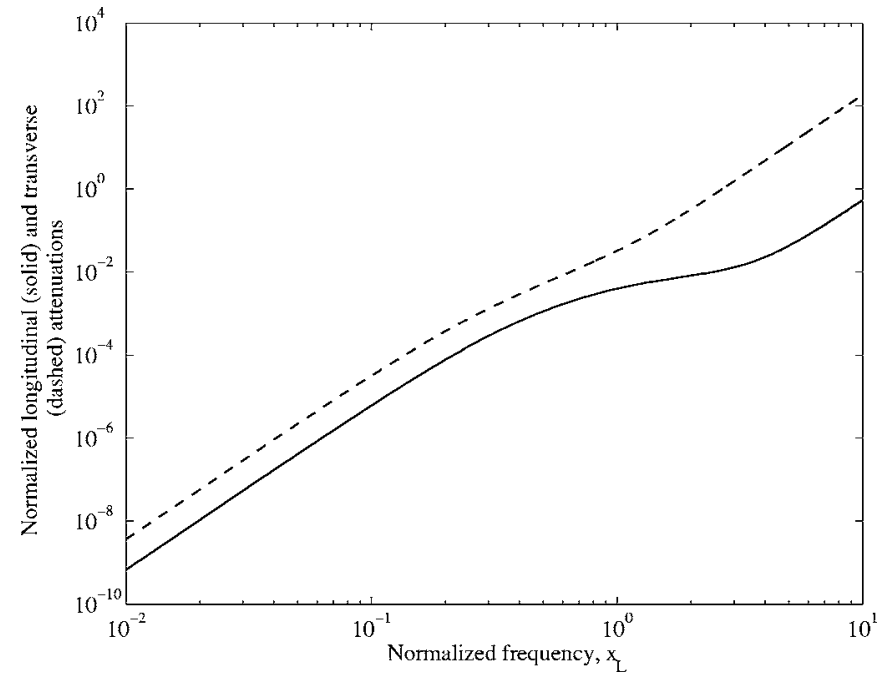

FIG. 11. Normalized longitudinal $\alpha_{L}$ and transverse $\alpha_{T}$ attenuations in terms of normalized frequency $x_{L}$.

Numerical results of the attenuations are now presented for titanium, using the single elastic constants given above. First, Fig. 11 presents the normalized longitudinal and transverse attenuations which are given in Eq. (21) as a function of dimensionless frequency, $x_{L}$, for the extreme case of statistically isotropy $(\sigma \rightarrow \infty)$. It is shown that the transverse attenuation is always greater than the longitudinal attenuation as shown in cubic crystals. Next, the general attenuations as a function of texture parameter $\sigma$ are presented. To compute the attenuations of three wave types, numerical integrations are employed in Eq. (20). The inner products on the covariance of the moduli fluctuations are obtained by Eq. (14). Using the covariance and wave propagation vectors obtained, the inner products of each wave type are calculated numerically. Then, the attenuations of the three wave types as a function of texture parameter $\sigma$ are obtained.

The texture dependence of the attenuations for a given dimensionless frequency $x_{S H}=0.5$ is presented. Figure 12 shows the dimensionless attenuation of the shear horizontal wave $(S H)$ as a function of the texture parameter $\sigma$ for given

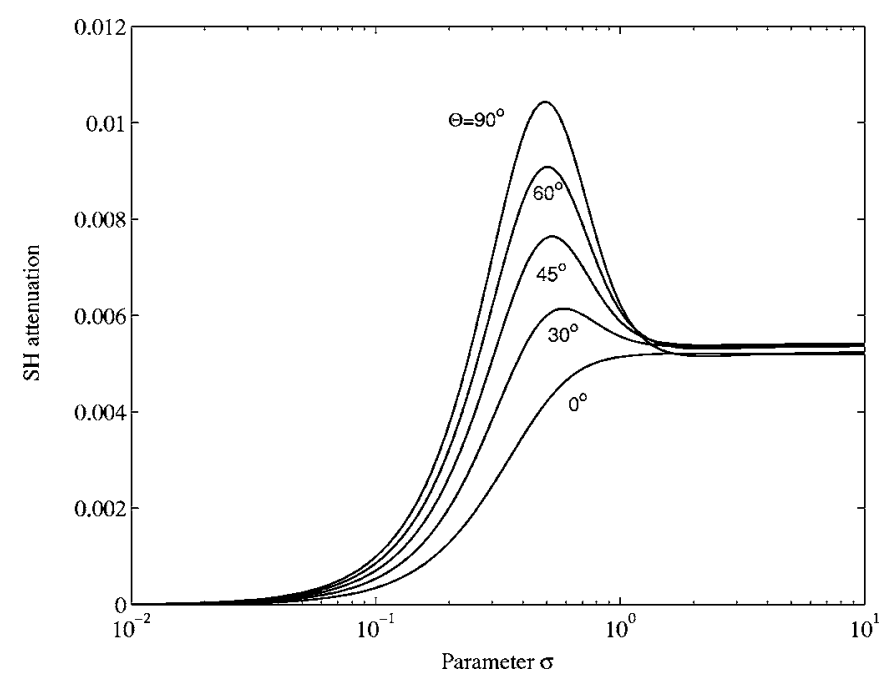

FIG. 12. Attenuations of the shear horizontal wave $(\mathrm{SH})$ versus texture parameter $\sigma$ with various wave propagation directions $\Theta$ and the given frequency $x_{S H}=0.5$.

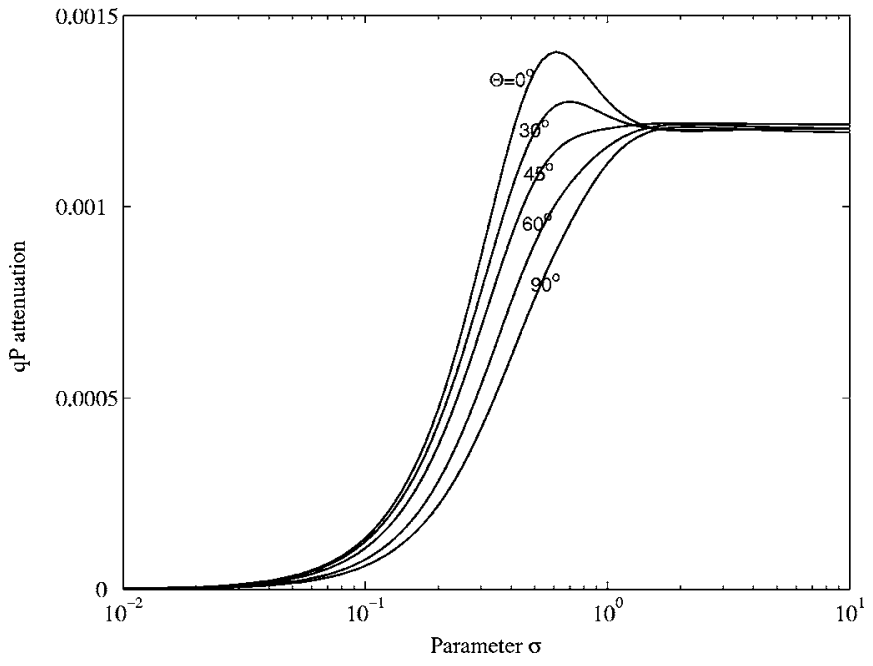

FIG. 13. Attenuations of the quasi-longitudinal wave $(q P)$ versus texture parameter $\sigma$ with various wave propagation directions $\Theta$ and the given frequency $x_{S H}=0.5$.

wave propagation directions $\Theta$. It is observed that attenuation is zero when the texture parameter $\sigma$ is small. Since in this limit the hexagonal crystals are perfectly aligned, they behave as a single crystal without scattering attenuation as expected. After the zero attenuation region, the attenuation increases to reach a maximum, then decreases to constant attenuation. The attenuations along various propagation directions all recover the isotropic limit as expected. The slight error observed in the isotropic limit is due to limits of the numerical integrations. It is also observed from Fig. 12 that there are different maxima for different wave propagation directions such that it is sensitive to the propagation direction due to texture. In particular, it is seen that there is no peak presented for $\Theta=0^{\circ}$. Such information is useful for monitoring microstructure evolution during processing.

In Fig. 13, the dimensionless quasi-longitudinal attenuation $(q P)$ is plotted versus the texture parameter $\sigma$ for the given dimensionless frequency and wave propagation direction. It is observed that the attenuation shows a similar track with texture parameter $\sigma$ to $S H$ wave as well. The attenuation is zero for $\sigma$ small, then increases to reach a maximum value. It is interesting to see that there are no maxima except at $\Theta=0^{\circ}$ and $\Theta=30^{\circ}$ appearing in other selected propagation directions. The dependence of these maxima on singlecrystal parameters is the subject of future research. The relationship between the attenuation of the quasi-shear vertical wave $(q S V)$ and the texture parameter $\sigma$ is presented in Fig. 14. It is seen that there are maxima displayed for some propagation directions. However, the variation of these peaks of the $q S V$ wave is much smaller than those of the $S H$ and $q P$ waves. Therefore, it might be concluded that the $S H$ and $q P$ waves are more sensitive to the grain orientation during processing. The attenuation results provide good motivation to use experiment measurements to detect changes of microstructure of hexagonal materials during the cold-working process. In addition, the experimental data could be used with various representative samples of cold working to clarify the peak observed in the theoretical predictions as part of future research. 


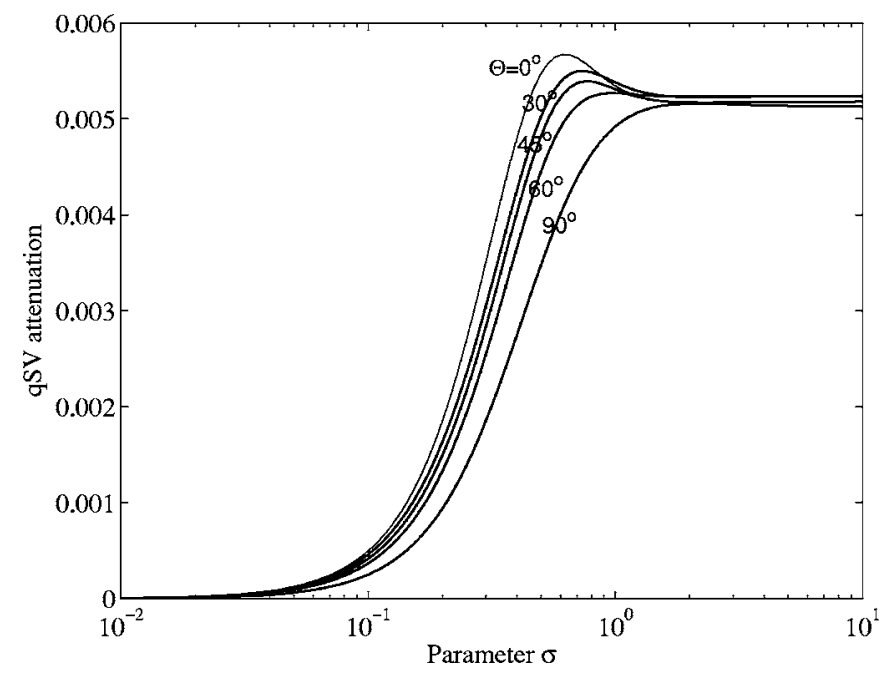

FIG. 14. Attenuations of the quasi-shear vertical wave $(q S V)$ versus texture parameter $\sigma$ with various wave propagation directions $\Theta$ and the given frequency $x_{S H}=0.5$.

\section{CONCLUSIONS}

In this article, the wave propagation and scattering in hexagonal polycrystalline materials with fiber texture was discussed. The quantitative relations between fiber texture and wave attenuations of hexagonal crystals were presented. The texture is characterized by a Gaussian distribution function that contains a single parameter that governs the transition of the texture from perfectly aligned crystals to statistically isotropic. Under this assumption, the materials of interest have a varying degree of transverse isotropy representative of processing conditions. Simple expressions for the attenuations of the three modes of waves are given in a concise representation. Finally, numerical results for titantium were presented and discussed in terms of the directional, frequency, and texture dependence. The results show that the attenuations of each wave type can be considerably affected during processing since the material's microstructure is changing. The results presented are expected to improve the understanding of the texture variations during processing. In experimental measurements, if one measures the attenuations at several times during the annealing process, it might be possible to obtain the texture and grain size information using this model, which is a future research topic.

\section{ACKNOWLEDGMENTS}

We gratefully acknowledge the financial support of the U.S. Department of Energy (Grant No. DE-FG0201ER45890).

\section{APPENDIX: EXAMPLE INNER PRODUCTS}

As an example, the inner products of the shear horizontal wave are given in the following. For the $S H$ wave, we have

$$
\begin{aligned}
\Xi_{\ldots \ldots \hat{\mathbf{u}}_{1} \hat{\mathbf{p}} \hat{\mathbf{p}} \hat{\mathbf{s}} \hat{\mathbf{v}}_{1}}= & \sin ^{2} \Theta \sin ^{2} \Theta^{\prime}\left[a_{1} \sin ^{4} \phi^{\prime}-a_{1} \sin ^{2} \phi^{\prime}+a_{2}\right]+a_{3} \\
& +\sin \Theta \cos \Theta \sin \Theta^{\prime} \cos \Theta^{\prime}\left[a_{4} \sin ^{3} \phi^{\prime}\right. \\
& \left.+a_{5} \sin \phi^{\prime}\right]+\cos ^{2} \Theta \cos ^{2} \Theta^{\prime}\left[a_{6} \sin ^{2} \phi^{\prime}+a_{7}\right]
\end{aligned}
$$

$$
\begin{aligned}
& \Xi \ldots \hat{\mathbf{u}}_{1} \hat{\mathbf{p}} \hat{\mathbf{s}} \hat{\mathbf{v}}_{2}=\sin ^{2} \Theta \sin ^{2} \Theta^{\prime} \sin ^{2} \gamma^{\prime}\left[a_{1} \sin ^{2} \phi^{\prime} \cos ^{2} \phi^{\prime}+a_{8}\right] \\
& +\sin ^{2} \Theta\left(\cos ^{2} \Theta^{\prime} \sin ^{2} \gamma^{\prime}+\sin ^{2} \Theta^{\prime} \cos ^{2} \gamma^{\prime}\right)\left[a_{4}\right] \\
& +\sin ^{2} \Theta \cos ^{2} \Theta^{\prime} \cos ^{2} \gamma^{\prime}\left[a_{9}\right] \\
& +\sin ^{2} \Theta \sin \Theta^{\prime} \cos \Theta^{\prime} \sin \gamma^{\prime} \cos \gamma^{\prime}\left[a_{10}\right] \\
& +\sin \Theta \cos \Theta\left(\cos ^{2} \Theta^{\prime} \sin \gamma^{\prime} \cos \gamma^{\prime}\right. \\
& \left.+\sin \Theta^{\prime} \cos \Theta^{\prime} \cos ^{2} \gamma^{\prime}\right)\left[a_{11} \sin \phi^{\prime}\right] \\
& +\sin \Theta \cos \Theta\left(\sin ^{2} \Theta^{\prime} \sin \gamma^{\prime} \cos \gamma^{\prime}\right. \\
& \left.+\sin \Theta^{\prime} \cos \Theta^{\prime} \sin ^{2} \gamma^{\prime}\right)\left[a_{12} \sin ^{3} \phi^{\prime}+a_{13} \sin \phi^{\prime}\right] \\
& +\cos ^{2} \Theta \cos ^{2} \Theta^{\prime} \cos ^{2} \gamma^{\prime}\left[a_{14}\right] \\
& +\cos ^{2} \Theta \sin ^{2} \Theta^{\prime} \sin ^{2} \gamma^{\prime}\left[a_{15} \cos ^{2} \phi^{\prime}+a_{16}\right] \\
& +\cos ^{2} \Theta\left(\sin ^{2} \Theta^{\prime} \cos ^{2} \gamma^{\prime}+\cos ^{2} \Theta^{\prime} \sin ^{2} \gamma^{\prime}\right) \\
& \times\left[a_{17} \cos ^{2} \phi^{\prime}+a_{18}\right] \\
& +\cos ^{2} \Theta \sin \Theta^{\prime} \cos \Theta^{\prime} \sin \gamma^{\prime} \cos \gamma^{\prime} \\
& \times\left[a_{19} \cos ^{2} \phi^{\prime}+a_{20}\right]
\end{aligned}
$$

$$
\begin{aligned}
\Xi_{\ldots . . . \hat{\mathbf{u}}_{1} \hat{\mathbf{p}} \hat{\mathbf{p}} \hat{\mathbf{s}} \hat{\mathbf{v}}_{3}}= & \sin ^{2} \Theta \sin ^{2} \Theta^{\prime} \cos ^{2} \gamma^{\prime}\left[a_{1} \sin ^{2} \phi^{\prime} \cos ^{2} \phi^{\prime}+a_{8}\right] \\
& +\sin ^{2} \Theta\left(\cos ^{2} \Theta^{\prime} \cos ^{2} \gamma^{\prime}+\sin ^{2} \Theta^{\prime} \sin ^{2} \gamma^{\prime}\right)\left[a_{4}\right] \\
& +\sin ^{2} \Theta \cos ^{2} \Theta^{\prime} \sin ^{2} \gamma^{\prime}\left[a_{9}\right] \\
& -\sin ^{2} \Theta \sin \Theta^{\prime} \cos \Theta^{\prime} \sin \gamma^{\prime} \cos \gamma^{\prime}\left[a_{10}\right] \\
& +\sin \Theta \cos \Theta\left(-\cos ^{2} \Theta^{\prime} \sin \gamma^{\prime} \cos \gamma^{\prime}\right. \\
& \left.+\sin \Theta^{\prime} \cos \Theta^{\prime} \sin ^{2} \gamma^{\prime}\right)\left[a_{11} \sin \phi^{\prime}\right] \\
& +\sin \Theta \cos \Theta\left(-\sin ^{2} \Theta^{\prime} \sin \gamma^{\prime} \cos \gamma^{\prime}\right. \\
& \left.+\sin \Theta^{\prime} \cos \Theta^{\prime} \cos ^{2} \sin ^{2} \gamma^{\prime}\right)\left[a_{12} \sin ^{3} \phi^{\prime}\right. \\
& \left.+a_{13} \sin \phi^{\prime}\right]+\cos ^{2} \Theta \cos ^{2} \Theta^{\prime} \cos ^{2} \gamma^{\prime}\left[a_{14}\right] \\
& +\cos ^{2} \Theta \sin ^{2} \Theta^{\prime} \cos ^{2} \gamma^{\prime}\left[a_{15} \cos ^{2} \phi^{\prime}+a_{16}\right] \\
& +\cos ^{2} \Theta\left(\sin ^{2} \Theta^{\prime} \sin ^{2} \gamma^{\prime}+\cos ^{2} \Theta^{\prime} \cos ^{2} \gamma^{\prime}\right) \\
& \times\left[a_{17} \cos ^{2} \phi^{\prime}+a_{18}\right] \\
& +\cos ^{2} \Theta \sin \Theta^{\prime} \cos ^{\prime} \sin ^{\prime} \cos ^{\prime} \\
& \times\left[a_{19} \cos ^{2} \phi^{\prime}+a_{20}\right]
\end{aligned}
$$

where the coefficients are given by

$$
\begin{aligned}
a_{1}= & -4\left(2 B M_{1}+D M_{3}\right)^{2}+32 B^{2} M_{3}+24 D^{2} N_{1} \\
& +48 B D M_{6}, \\
a_{2}= & -\left(2 B M_{1}+D M_{3}\right)^{2}+9 D^{2} N_{1}+8 B^{2} M_{3}+12 B D M_{6} \\
& -3 D^{2} N_{2}-B^{2} M_{4}-2 B D M_{7}, \\
a_{3}= & 3 D^{2} N_{2}+B^{2} M_{4}+2 B D M_{7}, \\
a_{4}= & -4 B D M_{2} M_{3}-8 B D M_{1} M_{3}-8 B D M_{1} M_{4} \\
& -4 D^{2} M_{3} M_{4}+12 B D M_{6}+16 B^{2} M_{3}+16 B^{2} M_{4} \\
& -8 B^{2} M_{1} M_{2}+36 B D M_{7}-16 B^{2} M_{1}^{2}+24 D^{2} N_{2},
\end{aligned}
$$

Yang et al:: Microstructure evolution during processing 


$$
\begin{aligned}
& a_{5}=-12 D^{2} N_{2}-22 B D M_{7}-6 B D M_{6}-8 B^{2} M_{3} \\
& +2 B D M_{2} M_{3}+4 B D M_{1} M_{3}+2 D^{2} M_{3} M_{4}+8 B^{2} M_{1}^{2} \\
& -10 B^{2} M_{4}+4 B^{2} M_{1} M_{2}+4 B D M_{1} M_{4} \text {, } \\
& a_{6}=B^{2} M_{5}-4 B^{2} M_{1}^{2}-D^{2} M_{4}^{2}+2 B^{2} M_{4}+4 B D M_{7} \\
& -2 B D M_{2} M_{4}-4 B D M_{1} M_{4}+2 D^{2} N_{3}+2 B^{2} M_{3} \\
& +2 B D M_{8}-4 B^{2} M_{1} M_{2}-B^{2} M_{2}^{2} \text {, } \\
& a_{7}=D^{2} N_{3}+B^{2} M_{3}-B^{2} M_{4}-3 D^{2} N_{2}, \\
& a_{8}=4 A B M_{3}+12 B D M_{6}+4 B^{2} M_{3}+6 A D M_{6}+A^{2} M_{3} \\
& +15 D^{2} N_{1} \\
& a_{9}=A^{2} M_{3}+2 A D M_{7}+D^{2} N_{3}, \\
& a_{10}=4 A B M_{3}+2 B^{2} M_{4}+8 B D M_{7}+6 A D M_{6}+2 A^{2} M_{3} \\
& +2 \mathrm{ADM}_{7}+12 \mathrm{D}^{2} \mathrm{~N}_{2}, \\
& a_{11}=2 A B M_{4}+4 D^{2} N_{3}+6 B D M_{7}+2 A B M_{3}+2 B D M_{8} \\
& +4 A D M_{7}+4 B^{2} M_{4}, \\
& a_{12}=-24 D^{2} N_{2}+16 B^{2} M_{1}^{2}-16 B^{2} M_{4}+8 B D M_{1} M_{4} \\
& -36 B D M_{7}+4 B D M_{2} M_{3}+8 B D M_{1} M_{3}+4 D^{2} M_{3} M_{4} \\
& -12 B D M_{6}+8 B^{2} M_{1} M_{2}-16 B^{2} M_{3} \text {, } \\
& a_{13}=36 D^{2} N_{2}-16 B^{2} M_{1}^{2}+16 B^{2} M_{4}+2 A B M_{3} \\
& -8 B D M_{1} M_{4}+42 B D M_{7}-4 B D M_{2} M_{3} \\
& -8 B D M_{1} M_{3}-4 D^{2} M_{3} M_{4}+2 A B M_{4}+18 B D M_{6} \\
& +4 A D M_{7}-8 B^{2} M_{1} M_{2}+20 B^{2} M_{3} \text {, } \\
& a_{14}=4 B D M_{8}+D^{2} N_{4}+A^{2} M_{4}+4 A B M_{4}+2 A D M_{8} \\
& +4 B^{2} M_{4} \\
& a_{15}=12 B D M_{7}+4 A B M_{4}+4 A D M_{7}+4 B^{2} M_{4}+12 D^{2} N_{2} \text {, } \\
& a_{16}=A^{2} M_{4}+2 A D M_{7}+3 D^{2} N_{2}, \\
& a_{17}=4 B D M_{7}+2 B D M_{8}-4 B D M_{1} M_{4}-2 B D M_{2} M_{4} \\
& -D^{2} M_{4}^{2}-4 B^{2} M_{1}^{2}+B^{2} M_{5}+2 B^{2} M_{4}+2 B^{2} M_{3} \\
& -4 B^{2} M_{1} M_{2}-B^{2} M_{2}^{2}+2 D^{2} N_{3} \text {, } \\
& a_{18}=2 B D M_{7}+B^{2} M_{3}+D^{2} N_{3}, \\
& a_{19}=16 B D M_{7}-8 B D M_{1} M_{4}-4 B D M_{2} M_{4}+8 B D M_{8} \\
& -2 D^{2} M_{4}^{2}-8 B^{2} M_{1}^{2}+4 B^{2} M_{3}-2 B^{2} M_{2}^{2}+4 A B M_{4} \\
& +4 A D M_{7}+2 B^{2} M_{5}+12 B^{2} M_{4}-8 B^{2} M_{1} M_{2} \\
& +8 D^{2} N_{3}
\end{aligned}
$$

$$
\begin{aligned}
a_{20}= & 8 B D M_{7}+2 B^{2} M_{3}+4 A B M_{4}+2 A^{2} M_{4}+2 A D M_{8} \\
& +2 A D M_{7}+4 D^{2} N_{3} ;
\end{aligned}
$$

with

$$
\begin{aligned}
& M_{6}=\left[\frac{1}{48}\left(I_{\sigma}^{0}-3 I_{\sigma}^{2}+3 I_{\sigma}^{4}-I_{\sigma}^{6}\right)\right], \quad M_{7}=\left[\frac{1}{8}\left(I_{\sigma}^{2}-2 I_{\sigma}^{4}+I_{\sigma}^{6}\right)\right], \\
& M_{8}=\left[\frac{1}{2}\left(I_{\sigma}^{4}-I_{\sigma}^{6}\right)\right], \quad M_{9}=I_{\sigma}^{6}, \\
& N_{1}=\left[\frac{1}{384}\left(I_{\sigma}^{0}-4 I_{\sigma}^{2}+6 I_{\sigma}^{4}-4 I_{\sigma}^{6}+I_{\sigma}^{8}\right)\right], \\
& N_{2}=\left[\frac{1}{48}\left(I_{\sigma}^{2}-3 I_{\sigma}^{4}+3 I_{\sigma}^{6}-I_{\sigma}^{8}\right)\right], \quad N_{3}=\left[\frac{1}{8}\left(I_{\sigma}^{4}-2 I_{\sigma}^{6}+I_{\sigma}^{8}\right)\right], \\
& N_{4}=\left[\frac{1}{2}\left(I_{\sigma}^{6}-I_{\sigma}^{8}\right)\right], \quad N_{5}=I_{\sigma}^{8},
\end{aligned}
$$

and $M_{i}(i=1,2,3,4,5)$ are given in Eq. (9). For the quasilongitudinal and quasi-shear waves, the inner products are not presented here due to brevity.

For the limiting case of statistical isotropy $(\sigma \rightarrow \infty)$, the inner products required to compute the attenuations given in Eq. (22) are expressed as

$$
\begin{aligned}
& \underset{\exists . . . \hat{\mathbf{p}} \hat{\mathbf{p}} \hat{\mathbf{p}} \hat{\mathbf{s}} \hat{\mathbf{s}}}{\mathbf{s}}=\left[\frac{4}{3} T_{4}+\frac{52}{15} T_{7}\right]+\left[4 T_{4}+\frac{64}{3} T_{5}+16 T_{6}\right. \\
& \left.+\frac{208}{15} T_{7}\right] \chi^{2}+\left[\frac{16}{3} T_{6}+\frac{28}{15} T_{7}\right] \chi^{4}, \\
& \Xi_{\ldots . \hat{\mathbf{p}} \hat{\mathbf{p}} \hat{\mathbf{s}} \hat{\mathbf{s}} \hat{\mathbf{s}}_{2}}=\Xi_{\ldots . \hat{\mathbf{p}}_{2} \hat{\mathbf{p}} \hat{\mathbf{s}} \hat{\mathbf{s}}}=\left[T_{4}+2 T_{7}\right]+\left[4 T_{5}+4 T_{6}+4 T_{7}\right] \chi^{2}, \\
& \Xi_{\ldots . \hat{\mathbf{p}} \hat{\mathbf{p}} \hat{\mathbf{p}} \hat{\mathbf{s}}_{3} \mathbf{s} \mathbf{s}_{3}}=\Xi_{\ldots . . \hat{\mathbf{p}}_{3} \hat{\mathbf{p}} \hat{\mathbf{p}} \hat{\mathbf{p}} \hat{\mathbf{s}} \hat{\mathbf{s}}}=\left[T_{4}+4 T_{5}+4 T_{6}+6 T_{7}\right]+\left[\frac{16}{3} T_{6}\right. \\
& \left.+\frac{28}{15} T_{7}\right] \chi^{2}+\left[\frac{16}{3} T_{6}+\frac{28}{15} T_{7}\right] \chi^{4}, \\
& \Xi_{\ldots . . \hat{\mathbf{p}}_{2} \hat{\mathbf{p}} \hat{\mathbf{p}} \hat{\mathbf{s}}_{2}} \hat{\mathbf{s}}_{2}=\left[4 T_{6}+4 T_{7}\right]+\left[T_{3}+3 T_{6}+2 T_{7}\right] \chi^{2}, \\
& \underset{\Xi \cdots \hat{\mathbf{p}}_{3} \hat{\mathbf{p}} \hat{\mathbf{s}}_{3}}{\hat{\mathbf{p}}_{3} \mathbf{\mathbf { s }} \hat{\mathbf{s}}_{3}}=\left[\frac{4}{3} T_{6}+\frac{52}{15} T_{7}\right]-\left[\frac{16}{3} T_{6}+\frac{28}{15} T_{7}\right] \chi^{2} \\
& +\left[\frac{16}{3} T_{6}+\frac{14}{15} T_{7}\right] \chi^{4}
\end{aligned}
$$

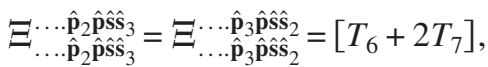

where

$$
\begin{aligned}
& T_{1}=-\frac{8}{45} A^{2}-\frac{16}{315} A D-\frac{16}{4725} D^{2}, \\
& T_{2}=-\frac{8}{315} A D-\frac{8}{315} B D-\frac{8}{45} A B-\frac{16}{4725} D^{2},
\end{aligned}
$$




$$
\begin{aligned}
& T_{3}=-\frac{8}{45} B^{2}-\frac{16}{315} B D-\frac{16}{4725} D^{2}, \\
& T_{4}=\frac{A^{2}}{15}+\frac{2}{105} A D+\frac{D^{2}}{945}, \\
& T_{5}=\frac{A B}{15}+\frac{A D}{105}+\frac{B D}{105}+\frac{D^{2}}{945}, \\
& T_{6}=\frac{B^{2}}{15}+\frac{2 B D}{105}+\frac{D^{2}}{945}, \\
& T_{7}=\frac{D^{2}}{945} .
\end{aligned}
$$

Ahmed, S., and Thompson, R. B. (1992). "Effects of preferred grain orientation and grain elongation on ultrasonic wave propagation in stainless steel," in Review of Progress in Quantitative NDE, Vol. 11, 1999-2006, edited by D. O. Thompson, and D. E. Chimenti (Plenum, New York).

Ahmed, S., and Thompson, R. B. (1996). "Propagation of elastic waves in equiaxed stainless-steel polycrystals with aligned [001] axes," J. Acoust.
Soc. Am. 99, 2086-2096.

Fedorov, A. F. (1968). Theory of Elastic Waves in Crystals (Plenum, New York).

Hirsekorn, S. (1982). "The scattering of ultrasonic waves by polycrystals," J. Acoust. Soc. Am. 72, 1021-1031.

Hirsekorn, S. (1983). "The scattering of ultrasonic waves by polycrystals. II. Shear waves," J. Acoust. Soc. Am. 73, 1160-1163.

Hirsekorn, S. (1985). "The scattering of ultrasonic waves in polycrystalline materials with texture," J. Acoust. Soc. Am. 77, 832-843.

Hirsekorn, S. (1986). "Directional dependence of ultrasonic propagation in textured polycrystals," J. Acoust. Soc. Am. 79, 1269-1279.

Li, J. Y. (2000). "The effective electroelastic moduli of textured piezoelectric polycrystalline aggregates," J. Mech. Phys. Solids 48, 529-552.

Li, Y., and Thompson, R. B. (1990). "Relations between elastic constants $C_{i j}$ and texture parameters for hexagonal materials," J. Appl. Phys. 67, 26632665.

Stanke, F. E., and Kino, G. S. (1984). "A unified theory for elastic wave propagation in polycrystalline materials," J. Acoust. Soc. Am. 75, 665681.

Turner, J. A. (1999). "Elastic wave propagation and scattering in heterogeneous, anisotropic media: Textured polycrystalline materials," J. Acoust. Soc. Am. 106, 541-552.

Weaver, R. L. (1990). "Diffusion of ultrasound in polycrystals," J. Mech. Phys. Solids 38, 55-86.

Yang, L., and Turner, J. A. (2004). "Attenuation of ultrasonic waves in rolled metals," J. Acoust. Soc. Am. 116, 3319-3327. 